\title{
Fast Pencil Beam Dose Calculation for Proton Therapy Using a Double- Gaussian Beam Model
}

\author{
Joakim da Silva ${ }^{1,2 *}$, Richard Ansorge ${ }^{1}$ and Rajesh Jena ${ }^{2}$ \\ ${ }^{1}$ Cavendish Laboratory, Department of Physics, University of Cambridge, Cambridge, UK, ${ }^{2}$ Department of Oncology, \\ University of Cambridge, Cambridge, UK
}

\section{OPEN ACCESS}

Edited by:

Marco Durante,

GSI, Germany

Reviewed by:

Dalong Pang,

Georgetown University Hospital, USA Andrea Fontana, Istituto Nazionale di Fisica Nucleare,

*Correspondence: Joakim da Silva jd491@cam.ac.uk

Specialty section: This article was submitted to Radiation Oncology, a section of the journal

Frontiers in Oncology

Received: 29 September 2015 Accepted: 30 November 2015 Published: 18 December 2015

Citation:

da Silva J, Ansorge $R$ and Jena $R$ (2015) Fast Pencil Beam Dose Calculation for Proton Therapy Using a Double-Gaussian Beam Model.

Front. Oncol. 5:281. doi: 10.3389/fonc.2015.00281
The highly conformal dose distributions produced by scanned proton pencil beams (PBs) are more sensitive to motion and anatomical changes than those produced by conventional radiotherapy. The ability to calculate the dose in real-time as it is being delivered would enable, for example, online dose monitoring, and is therefore highly desirable. We have previously described an implementation of a PB algorithm running on graphics processing units (GPUs) intended specifically for online dose calculation. Here, we present an extension to the dose calculation engine employing a double-Gaussian beam model to better account for the low-dose halo. To the best of our knowledge, it is the first such PB algorithm for proton therapy running on a GPU. We employ two different parameterizations for the halo dose, one describing the distribution of secondary particles from nuclear interactions found in the literature and one relying on directly fitting the model to Monte Carlo simulations of PBs in water. Despite the large width of the halo contribution, we show how in either case the second Gaussian can be included while prolonging the calculation of the investigated plans by no more than $16 \%$, or the calculation of the most time-consuming energy layers by about $25 \%$. Furthermore, the calculation time is relatively unaffected by the parameterization used, which suggests that these results should hold also for different systems. Finally, since the implementation is based on an algorithm employed by a commercial treatment planning system, it is expected that with adequate tuning, it should be able to reproduce the halo dose from a general beam line with sufficient accuracy.

Keywords: pencil beam, proton therapy, dose calculation, double Gaussian, graphics processing unit, adaptive radiotherapy

\section{INTRODUCTION}

Fast dose calculation finds use in a variety of radiotherapy applications and is an active area of research (1). Due to the high level of dose conformity, the small number of treatment fields, and the sensitivity to material changes in the beam path, adaptive treatment techniques relying on fast, repeated dose calculation are of particular interest in proton therapy. A considerable amount of work has therefore gone into using graphics processing units (GPUs) to speed up proton therapy Monte Carlo (MC) dose calculation in order to allow daily dose recalculation (2-6). However, more advanced adaptive techniques, such as real-time dose monitoring, would involve calculating the dose online as it is being delivered. For a treatment employing pencil beam (PB) scanning, this 
would require the calculation time of individual energy layers to be short in comparison with the time between energy layers or the length of a typical motion phase. For such applications, GPU $\mathrm{MC}$ dose calculation on a single workstation remains too slow by at best one, and generally two or more, orders of magnitude. In a previous paper, we therefore presented a GPU implementation of the widely used PB algorithm, especially developed for use in online calculation (7). The presented dose calculation engine was capable of calculating a two-field, base-of-skull test case in $0.22 \mathrm{~s}$, with individual energy layers of the same case taking $6.4 \mathrm{~ms}$ or less to calculate. The short calculation times were largely attributed to the efficient GPU implementation of the computationally expensive kernel superposition (KS) step of the algorithm (8). Although the accuracy of the calculation in the high- and medium-dose regions was seen to be high, with $\gamma$-index passing rates matching those of a PB algorithm in clinical use, the implementation used a single-Gaussian kernel to describe the lateral dose profiles of PBs. It is well-known that such a beam model cannot accurately predict the low-dose halo made up of particles traveling at large angles with the beam direction, originating from nuclear interactions, inhomogeneous scattering in the nozzle, or large-angle Rutherford scattering. Despite the low halo dose, their large widths mean that the halos from a number of PBs may overlap to produce a noticeable impact on the overall dose distribution. Modeling of the halo dose is therefore necessary to predict the field-size dependence of the central dose in energy layers. In addition, the halos are responsible for the low-dose region further away from the target, which might be of interest when trying to predict the risk of developing side effects or secondary tumors.

Although PB algorithms for proton therapy have traditionally employed the single-Gaussian beam model, the above reasons have led to modern treatment planning systems (TPSs) almost exclusively making use of more complex models. Generally, these add one or more additional terms for modeling the halo dose to the Gaussian kernel describing the contribution from primary particles. A common method is to use a second, wider Gaussian to describe the halo, an approach first suggested for dose calculation in electron therapy (9). The potential to use the same approach in proton therapy was later pointed out in a paper describing the implementation of a commercial TPS (10). Pedroni et al. presented the first implementation of a doubleGaussian beam model for scanned PBs, using a parameterization based on measurements of the increase in central dose in square fields of increasing side length (11). Later the same year, Soukup et al. presented a different implementation, where the parameterization was instead based on MC simulations of nuclear interactions in water, which was adopted in the commercial TPS XiO Proton (Elekta AB, Stockholm, Sweden) (12). Since then a range of parameterizations, based on measurements, MC simulations, and analytical calculations, have been presented (13-17). Furthermore, several extensions to the double-Gaussian model have been suggested, including using a double-Gaussian model also for the PB shape in air, adding a third Gaussian to the beam model, and adding different non-Gaussian functions to the kernel (18-24).

From the above discussion, it is clear that to be fully comparable to a modern TPS, a calculation engine for online dose monitoring must include a model for the halo dose. Starting with an existing implementation, a double-Gaussian beam model could easily be implemented by simply rerunning the calculation a second time for the halo contribution. The difficulty in a fast implementation, however, stems from the width of the halo: for a double-Gaussian beam model, the width of the halo contribution is expected to be about two to three times larger than that of the primary contribution. The most time-consuming step of the $\mathrm{PB}$ algorithm is the KS, where the computational PBs (CPBs) - the computational elements of the $\mathrm{PB}$ algorithm obtained from the sub-PB splitting of physical PBs (7) - are widened perpendicular to the beam direction through a superposition of the kernels describing their lateral shape. The number of voxels reached by the kernel at a given depth, and thereby the calculation time of the KS step, is proportional to the square of the CPB width and inversely proportional to the square of the CPB spacing. Therefore, the calculation of a two to three times wider halo dose is expected to take four to nine times longer than that of the primary contribution, threatening to make the calculation time prohibitively long for real-time applications. Here, we describe the integration of a double-Gaussian beam model, based on the algorithm by Soukup et al. (12), into our previously presented GPU dose calculation engine, which aims to reduce the calculation time of the halo dose. It does so in part by employing the method described in Ref. (12), where, in the calculation of the halo dose, a single "nuclear" $\mathrm{PB}$, henceforth referred to as halo $\mathrm{PB}$ (HPB), is assigned to each physical PB. Assuming $3 \mathrm{~mm} \mathrm{~PB}$ spacing and $1 \mathrm{~mm} \mathrm{CPB}$ spacing for the primary contribution, this lack of sub-PB splitting reduces the number of HPBs, and thus their computational load, by a factor of nine. In addition, we employ a separate beam's-eye-view (BEV) coordinate system for the halo dose. This is defined in the same way as the $\mathrm{CPB}$ coordinate system described in our previous implementation (7) but is based on the HPB grid spacing. Using the same assumption as above, this effectively reduces the resolution of the halo calculation by a factor of three, and thereby the computational load of the KS by another factor of nine. Thus, using this approach, the time required by the KS step for the HPBs is expected to be not more than $11 \%$ of that for the primary CPBs. Although the main focus of the work was the implementation of the double-Gaussian beam model and its performance, it also includes a comparison of two parameterization approaches for the double-Gaussian beam model, so that their effect on the calculation time could be investigated.

\section{MATERIALS AND METHODS}

\section{Algorithm}

The PB implementation presented in this paper assumes that the dose $D$ to a point $(x, y, z)$ can be described by a double-Gaussian beam model according to

$$
\begin{aligned}
\mathrm{D}(x, y, z)= & \sum_{\mathrm{i} \in \mathrm{CPB}}\left(1-u\left(E_{\mathrm{i}}, z_{\mathrm{WE}, \mathrm{i}}\right)\right) \times N_{\mathrm{i}} \times I_{\mathrm{IDD}}\left(E_{\mathrm{i}}, z_{\mathrm{WE}, \mathrm{i}}\right) \\
& \times G\left(x-x_{\mathrm{i}}, y-y_{\mathrm{i}}, \sigma_{\mathrm{CPB}, \mathrm{i}}\right)+\sum_{\mathrm{j} \in \mathrm{HPB}} u\left(E_{\mathrm{j}}, z_{\mathrm{WE}, \mathrm{j}}\right) \\
& \times N_{\mathrm{j}} \times I_{\mathrm{IDD}}\left(E_{\mathrm{j}}, z_{\mathrm{WE}, \mathrm{j}}\right) \times G\left(x-x_{\mathrm{j}}, y-y_{\mathrm{j}}, \sigma_{\mathrm{HPB}, \mathrm{j}}\right)
\end{aligned}
$$


The first sum on the right-hand side of Eq. 1 represents the dose from primary particles, which is calculated according to the original implementation using the single-Gaussian beam model presented elsewhere (7). Consequently, the index of summation, $i$, runs over the CPBs resulting from the sub-PB splitting of the physical PBs, and each factor inside the summation (except for the first one) is further identical to what was previously presented. Specifically, $N_{\mathrm{i}}$ is the CPB weight, $E_{\mathrm{i}}$ is the initial beam energy, $z_{\mathrm{WE}, \mathrm{i}}$ is the water-equivalent path length (WEPL), $I_{\text {IDD }}$ is the integral depth dose (IDD), $G$ is the Gaussian function, and $\sigma_{\mathrm{CPB}, \mathrm{i}}$ is the SD of the primary Gaussian, henceforth referred to as the CPB width. In Eq. $1, u\left(E_{\mathrm{i}}, z_{\mathrm{WE}, \mathrm{i}}\right) \in[0,1]$ is the halo fraction, defined as the share of the integral dose that is deposited by the halo, which is given by the halo dose parameterization. Consequently, the factor $\left[1-u\left(E_{\mathrm{i}}, z_{\mathrm{WE}, \mathrm{i}}\right)\right]$ gives the fraction of the integral dose at a given WEPL that is deposited by primary particles. It should be noted that, although the CPB widths are calculated as described in the original publication, the values of the parameters $E_{\mathrm{S}}$ and $\delta$, which enter the width calculation as free parameters in the implementation, have to be adjusted in the double-Gaussian beam model. This is because the values (14.1 MeV and $0.21 \mathrm{~mm}$, respectively) obtained for the single-Gaussian beam model were based on how well the shape of the calculated PBs reproduced the total dose distributions, including contributions from the low-dose halo, from individual PBs as obtained by MC simulations. When employing the double-Gaussian model, these should instead be determined by a fit to the primary contribution alone. Therefore, the contribution from the halo should be subtracted from the total dose before finding the best values of $E_{\mathrm{S}}$ and $\delta$, and these must therefore be determined separately for each halo dose parameterization.

The dose contribution from the low-dose halo is given by the second sum on the right-hand side of Eq. 1. In this case, the sum is taken over HPBs, which, since no sub-PB splitting is applied, coincide in number and position with the physical PBs. Therefore, the weight, $N_{\mathrm{j}}$, of $\mathrm{HPB} \mathrm{j}$ is equal to that of the corresponding physical PB. The width of HPB j, $\sigma_{\mathrm{HPB}, \mathrm{j}}$, is defined in accordance with Ref. (12) as

$$
\sigma_{\mathrm{HPB}}(E, z)=\sqrt{\sigma_{\mathrm{PB}}^{2}(E, z)+\sigma_{\mathrm{LA}}^{2}\left(E, z_{\mathrm{WE}}\right)}
$$

where $\sigma_{\mathrm{PB}}$ is the total width of the contribution from primary protons before the sub-PB splitting, and $\sigma_{\mathrm{LA}}$ is the large-angle component given by the halo dose parameterization. Similar to $u$, and contrary to $\sigma_{\mathrm{CPB}}$ (and thus $\sigma_{\mathrm{PB}}$ ), $\sigma_{\mathrm{LA}}$ depends only on the initial beam energy and the WEPL.

\section{Beam Model Parameterizations}

Two different parameterizations for the halo fraction, $u$, and the large-angle component, $\sigma_{\mathrm{LA}}$, were investigated. The first parameterization, which will be referred to as the Soukup model, makes use of the unmodified analytical fits to MC data of nuclear interaction products given in Ref. (12), given by

$$
\begin{aligned}
u\left(E, z_{\mathrm{WE}}\right)= & 0.052 \log \left(1.13+\frac{z_{\mathrm{WE}}}{11.2-0.023 R_{0}(E)}\right) \\
& +0.35 \frac{0.0017 R_{0}^{2}(E)-R_{0}(E)}{\left(R_{0}(E)+3\right)^{2}-z_{\mathrm{WE}}^{2}}-1.61 \times 10^{-9} \\
& \times z_{\mathrm{WE}} \times\left(R_{0}(E)+3\right)^{2}
\end{aligned}
$$

where, if the right-hand side becomes negative, $u\left(E, z_{\mathrm{WE}}\right)$ is set to 0 , and

$$
\begin{aligned}
& \sigma_{\mathrm{LA}}\left(E, z_{\mathrm{WE}}\right)=2.85+0.0014 R_{0}(E) \times \log \left(z_{\mathrm{WE}}+3\right) \\
& +0.06 z_{\mathrm{WE}}-7.4 \times 10^{-5} \times z_{\mathrm{WE}}^{2}-0.22 \frac{R_{0}(E)}{\left(z_{\mathrm{WE}}-R_{0}(E)-5\right)^{2}}
\end{aligned}
$$

In the above equations, $R_{0}(E)$ is the Bragg peak $(\mathrm{BP})$ depth in water for a $\mathrm{PB}$ of initial energy $E$. Past the BP, both $u$ and $\sigma_{\mathrm{LA}}$ are assumed to take the same value as at the BP depth (although this is only stated explicitly for $u$ in the original publication). In order to calculate the new values for $E_{\mathrm{S}}$ and $\delta$ for the primary contribution, the radial halo distributions of individual $\mathrm{PBs}$ in water were calculated using the expression inside the second sum on the right-hand side of Eq. 1 together with Eqs 3 and 4 . The results were then subtracted from the corresponding radial dose distributions obtained from $\mathrm{MC}$ simulations to obtain the expected radial dose distribution of the primary particles. For each depth and energy, these were then fitted with a Gaussian function to extract the values for $\sigma_{\mathrm{PB}}$ in water. Since $\sigma_{\mathrm{HPB}}$ in Eq. 1 itself depends on $\sigma_{\mathrm{PB}}$, this process was done iteratively, using $\sigma_{\mathrm{PB}}$ from the singleGaussian implementation as the starting point. However, due to $\sigma_{\mathrm{LA}}$ generally being at least a factor of two larger than $\sigma_{\mathrm{PB}}$, the exact value of the latter was seen to play a limited role, resulting in the calculation converging after a single iteration. The resulting values of $\sigma_{\mathrm{PB}}$ were finally used to obtain the new values for the parameters $E_{\mathrm{S}}$ and $\delta$ in the same way as for the single-Gaussian beam model (7).

The second parameterization, which will be referred to as the direct model, relied on fitting sums of two Gaussians directly to the total radial dose distributions obtained from MC simulations, similar to Parodi et al. (17). This was done through a non-linear least squares fit using the trust-region algorithm provided with the Optimization Toolbox of Matlab (Mathworks, Natick, MA, USA). Despite the fact that the radial dose distributions quickly become very small for large radii, the contributions at large radii are important for two reasons. First, the radial distributions do not reflect the larger volumes receiving the contributions from larger radii, which is part of the reason why the low-dose halo is of interest in the first place. Second, since the dose fraction of the halo is expected to be smaller, but the width of its Gaussian larger than for the primary particles, its dose contribution will be dwarfed close to the central axis, and its parameters must thus be determined mainly from the dose at large radii. Therefore, in order for the optimization not to ignore the 
small contributions at large radii, the contribution to each radial bin was weighted according to the total area of a ring of the same width, i.e., with $\pi\left(r_{i+1}^{2}-r_{i}^{2}\right)$ for the bin between radius $r_{\mathrm{i}}$ and $r_{\mathrm{i}+1}$. The fit allowed the three parameters $\sigma_{\mathrm{PB}}$, $\sigma_{\mathrm{HPB}}$, and $u$ to be obtained simultaneously for each PB energy and depth in water. Values of $\sigma_{\mathrm{LA}}$ for different depths were then obtained from $\sigma_{\mathrm{PB}}$ and $\sigma_{\mathrm{HPB}}$ by rearranging Eq. 2 . In order to reduce the noise present in the calculated depth curves for $u$ and $\sigma_{\mathrm{LA}}$ (c.f. Figure 2), these were fitted with cubic splines to obtain the final parameterizations. Past the BP, where few charged particles remain in the beam, the basis for using separate Gaussians for charged primaries and secondaries starts to break down. This was characterized by a sharp drop in $u$ (likely due to the charged secondaries stopping earlier than primaries), followed by $u$ tending toward unity, while $\sigma_{\mathrm{LA}}$ grows very large, consistent with the figures shown in Ref. (17). The behavior is likely explained by the limited number of charged particles past the BP causing the second Gaussian to be fitted to the "aura" of uncharged secondaries (24), which is more appropriately described by a non-Gaussian function (20, $21,23,24)$. Although a double-Gaussian fit still provides some improvement in this region (c.f. bottom row of Figure 1), the aura was ignored, consistent with our approach at shallower depths. Thus, past $102 \%$ of the BP depth, the same solution of keeping the values of $u$ and $\sigma_{\mathrm{LA}}$ constant as for the Soukup model was employed. The values of $E_{S}$ and $\delta$ were finally obtained from $\sigma_{\mathrm{PB}}$ in the same way as before.

\section{Implementation}

Incorporating the double-Gaussian beam model in Eq. 1 into the existing $\mathrm{PB}$ dose calculation engine could in theory be achieved by carrying out the same calculation procedure twice: once for the primary and once for the halo contributions. However, there are two strong arguments against this solution. First, several parts of the implementation rely on assigning one thread per lateral voxel position. While this works well for the large number of $\mathrm{CPBs}$ used to calculate the primary contribution, the number of HPBs is expected to be about nine times lower since no sub-PB splitting is applied. Therefore, small- and medium-sized treatment fields would likely not contain enough HPBs to saturate a modern GPU. Second, several of the intermediates and results obtained in the calculation of the primary contribution, importantly the WEPL and $\sigma_{\mathrm{CPB}}$ (from which $\sigma_{\mathrm{PB}}$ is obtained), are also needed to calculate the halo contribution. Therefore, repeating the whole calculation would either require recalculating the necessary intermediates or keeping large amounts of data in global memory between the two rounds of calculations. Instead, it was deemed more efficient to maintain the structure presented in the original publication [c.f. Figure 2 in Ref. (7)] for the calculation of the primary contribution and to interleave it with the halo dose calculation. While the calculation of primary dose thus remains identical to what was previously presented, the following paragraph describes the changes made to the dose calculation engine in order to accommodate the halo dose calculation.

The only part of the implementation that was significantly modified compared to the original was the one calculating and storing the integral dose and kernel parameter for the $\mathrm{CPBs}$, which was extended to perform the same operations for the HPBs as well. Due to the smaller number of HPBs than CPBs, the additional operations were carried out only by the threads corresponding to CPBs whose positions coincide with that of a HPB. Although it led to the majority of threads being idle during the additional operations, this method was deemed preferable compared to using a separate calculation step for the HPBs, which would in any case not have enough parallelism to saturate the GPU. For each step along the $z$-axis, the widths of the HPBs were calculated according to Eq. 2. The required value of $\sigma_{\mathrm{LA}}^{2}$ was found by linear interpolation into a $2 \mathrm{D}$ texture containing the selected parameterization, and $\sigma_{\mathrm{PB}}^{2}$ was calculated by adding in quadrature the $\mathrm{PB}$ width at the patient surface, $\sigma_{\text {air }}\left(E, z_{0}\right)$, to the value of $\sigma_{\mathrm{CPB}}$ already calculated for the primary contribution. Furthermore, the integral dose for the halo contribution was obtained by multiplying the local IDD contribution, the full PB weight, and $u$, where $u$ was again obtained by interpolating into a $2 \mathrm{D}$ texture. (A multiplication with $(1-u)$ was similarly introduced in the calculation of the integral dose for the primary contribution.) To be compatible with the efficient KS implementation presented in a previous paper, the obtained values for the integral voxel dose and kernel parameter were then converted to dimensionless voxel units (8). These values were stored in two additional global memory arrays alongside those of the primary contribution. The KS step of the halo dose was identical to that of the primary contribution, and the two KS steps were carried out sequentially for each energy layer. However, due to the different resolutions of the CPB and HPB BEV systems, the BEV halo dose was kept in a separate $\mathrm{BEV}$ dose array. For the same reason, the transformation of the BEV halo dose to the global dose grid after completing the calculation for all energy layers also had to be done separately from the BEV primary dose.

\section{Validation and Benchmarking}

The double-Gaussian beam model was validated and benchmarked in the same way as the original dose calculation engine (7). In brief, all the reference dose distributions were obtained using the Fluka MC code, employing the beam line parameters and nozzle geometry of the Centro Nazionale di Adroterapia Oncologica (CNAO) treatment center in Pavia, Italy (25, 26). For the single $P B$ validation, the radial dose distribution from a $\mathrm{PB}$ of $\mathrm{BP}$ depth $220 \mathrm{~mm}$ in water was calculated using $1 \mathrm{~mm} C P B$ spacing and a global dose grid resolution of $1 \mathrm{~mm} \times 1 \mathrm{~mm} \times 1 \mathrm{~mm}$ and was compared with the corresponding reference $\mathrm{PB}$. For patient case validation, a base-of-skull plan for a $55.6 \mathrm{~cm}^{3}$ planning tumor volume target consisting of two oblique fields of 38 and 45 energy layers was used. The PB spacing (and thus HPB spacing) was $3 \mathrm{~mm}$, the CPB spacing was again set to $1 \mathrm{~mm}$, and, in order to match the resolution of the provided reference MC simulation, a global dose resolution of $2 \mathrm{~mm} \times 2 \mathrm{~mm} \times 2 \mathrm{~mm}$ was used. The same patient case was also used in the benchmarking. In addition, benchmarking was carried out on a plan for a cubic target of side length $100 \mathrm{~mm}$ extending 100-200 mm below the surface of a water tank and 

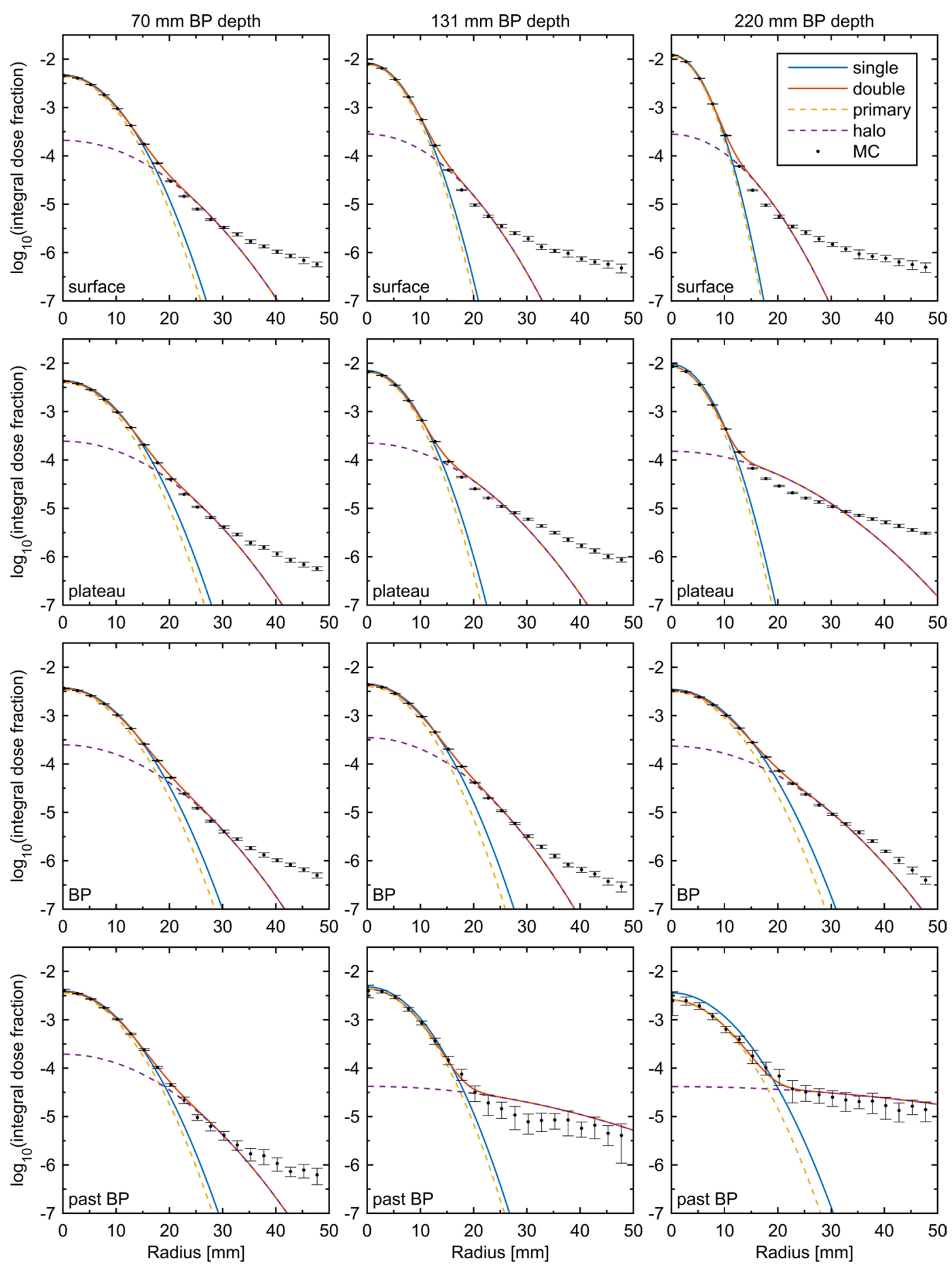

FIGURE 1 | Radial dose profiles as simulated by MC (black dots with error bars) fitted using single- and double-Gaussian models (solid lines). Dashed lines show the components of the double-Gaussian model. The error bars correspond to three times the estimated SD from the MC simulation, and to avoid crowding only every fifth MC data point is shown. Columns, from left to right, correspond to PBs with BP depths 70, 131, and 220 mm. Rows, from top to bottom, correspond to the profiles at the surface, at $40 \%$ of the BP depth, at the BP depth, and at $104 \%$ of the BP depth. The legend of the top left panel applies to all panels.

consisting on 20 energy layers. For this plan, the PB spacing was $3 \mathrm{~mm}$, the $\mathrm{CPB}$ spacing was set to $1 \mathrm{~mm}$, and a global dose resolution of $1 \mathrm{~mm} \times 1 \mathrm{~mm} \times 1 \mathrm{~mm}$ was used. The calculations were carried out on a Tesla K40 GPU from Nvidia (Santa Clara, CA, USA) with 2880 cores running at a clock frequency of $875 \mathrm{MHz}$. 

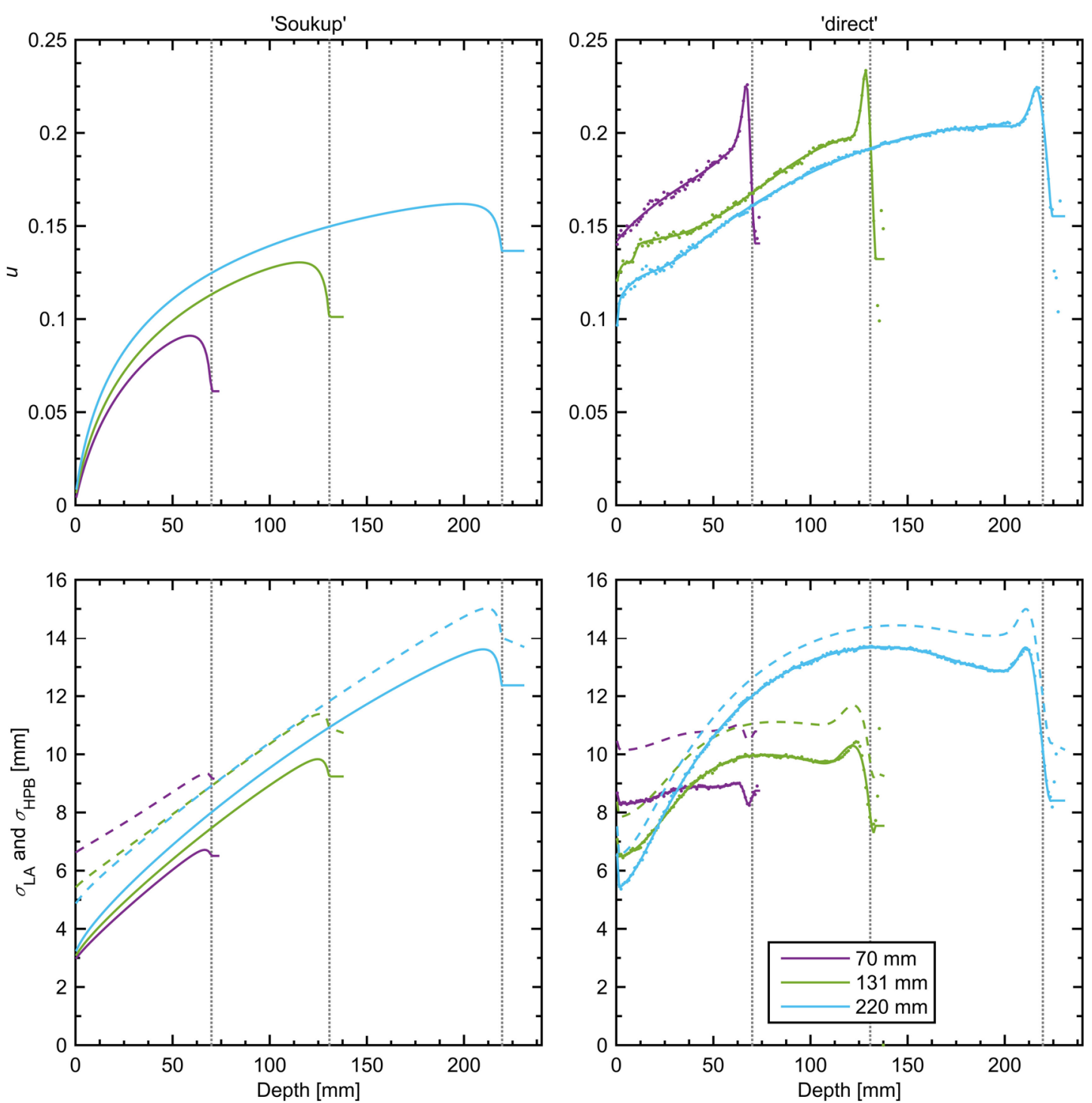

FIGURE 2 | Halo fraction, $u$, of the total dose (top row), $\sigma_{\mathrm{LA}}$ (bottom row, solid lines), and $\sigma_{\mathrm{HPB}}$ in water (bottom row, dashed lines) for the two parametrizations at three different beam energies. The individual data points used to fit the splines of the direct model are shown as dots (frequently coinciding with the corresponding line) in the right-hand panels, making visible the oscillating behavior in the model past $102 \%$ of the BP depth (with some dots falling outside the panels). Data for each parameterization are shown until 105\% of the BP depth, the largest WEPL considered in the calculation. The BP depth for each line color is given by the legend in the bottom right panel and is also indicated by vertical dotted lines.

\section{RESULTS}

\section{Beam Model Parameterizations}

The results of directly fitting a sum of two Gaussians to the radial profiles of $\mathrm{PBs}$ of three different energies in water are shown in Figure 1. Each $\mathrm{PB}$ is shown at four depths, corresponding to the surface, $40 \%$ of the BP depth, the full BP depth, and 104\% of the BP depth, and for comparison direct fits using a single-Gaussian are shown. A clear deviation from a single Gaussian is seen for all beam energies and at all depths, including the surface, indicating that nuclear interactions may not be the only factor contributing to the low-dose halo in the beam line modeled. For larger radii, it is clear that even an ideal double-Gaussian model breaks down far away from the central axis. However, for all depths up until just after the BP, this happens at a dose level, which is at least one order of magnitude smaller than for the single-Gaussian model.

Figure 2 shows curves of $u$ and $\sigma_{\mathrm{LA}}$ according to the two parameterizations considered and for three beam energies with BPs at 70, 131 , and $220 \mathrm{~mm}$ depth in water. A striking feature of this figure is the large difference in the shapes and magnitudes of both $u$ and $\sigma_{\text {LA }}$ between the models, especially at low beam energies. This shows that the assumptions made in the parameterization do indeed impact the resulting beam model. Although it was hard to perform a quantitative comparison with the data shown by Parodi et al. (17), the shapes and magnitudes of their curves for the CNAO treatment center seem to agree with those of the direct model presented here, 
as expected from the similar method used. The direct model also shows the closest agreement with the measurement-based parameterization by Pedroni et al. in terms of the shapes of the curves for $u$ and $\sigma_{\mathrm{HPB}}$, although their values of $u$ were seen to be almost half the size, and their values of $\sigma_{\mathrm{HPB}}$ a few millimeters larger (11). In addition to the differences in $u$ and $\sigma_{\mathrm{LA}}$, a difference was also seen in the new values of $E_{\mathrm{S}}$, which were $13.8 \mathrm{MeV}$ for the Soukup model and $13.0 \mathrm{MeV}$ for the direct model. The new values of the empirical correction $\delta$ were, in the same order, 0.00 and $0.06 \mathrm{~mm}$. The low values are expected since any major deviations from the multiple-scattering model should now be incorporated in the halo contribution. For the Soukup model, the halo fraction is close to 0 at the surface, which means that the width of the primary contribution at the surface should be given by the total PB width in air, as was the case for the single-Gaussian beam model. However, as can be seen from Figure 2, the halo fraction at the surface is non-zero for the direct model. The calculated PB width in air at the surface thus corresponds to the effective width of the primary and halo contributions taken together, and subsequently the width of the primary contribution must be smaller than this. It was seen that, in order to obtain the correct total beam width at the entrance point, the width of the primary contribution had to be set $2-4 \%$ smaller than the calculated PB width in air across the different energies. Therefore, when using the direct model, the entry width used in the calculation of the weights for the primary CPBs was set to $97 \%$ of the width calculated in air.

\section{Validation}

Figure 3 shows the difference in calculated dose for a PB of $220 \mathrm{~mm}$ $\mathrm{BP}$ depth when comparing the presented dose calculation engine using different halo dose parameterizations with the reference MC simulation. The result obtained for the previously presented singleGaussian beam model is included for reference, showing that both parameterizations considerably reduce the average error in comparison. Unsurprisingly, the smallest average error was achieved for the direct model, with the Soukup model performing surprisingly well despite the lack of beam line-specific tuning. The error ranges in Figure 3 were -0.8 to 2.1 and -1.8 to $2.0 \%$, respectively, for the Soukup and direct models, compared to -1.1 to $5.3 \%$ for the single-Gaussian model. The small value of the lower boundary of the direct model was caused by the large underestimation seen along the central axis close to the surface in Figure 3.

Figure 4 shows $\gamma$-index maps according to the $2 \% / 2 \mathrm{~mm}$ criterion for the reference patient case. Although the $\gamma$-index is a poor measure of the agreement in the low-dose region, better modeling of the halo dose is expected to be somewhat reflected in the $\gamma$-index due to the contribution of overlapping halos from multiple PBs to the high- and medium-dose regions. The $\gamma$-index passing rates for voxels receiving at least $10 \%$ of the prescription dose according to the $2 \% / 2 \mathrm{~mm}$ criterion were $97.9 \%$ for the Soukup model and $97.4 \%$ for the direct model, compared to $96.7 \%$ for the single-Gaussian model. For the less strict 3\%/3 mm criterion, the passing rates for the Soukup and direct models were 99.4 and $99.2 \%$, respectively, similar to the $99.2 \%$ obtained for the single-Gaussian model.

\section{Benchmarking}

Despite the differences seen in Figure 2, the performance of the two parameterizations of the double-Gaussian beam model was very similar. The calculation times for the patient case were 241 and $244 \mathrm{~ms}$, respectively, for the Soukup and direct models. This constitutes increases in the calculation time of 8 and $9 \%$ compared to the $224 \mathrm{~ms}$ required by the single-Gaussian model. The increase in calculation time for individual energy layer was seen to be larger and shifted toward smaller energy layers: the shortest calculation time (excluding memory transfers and deallocations)

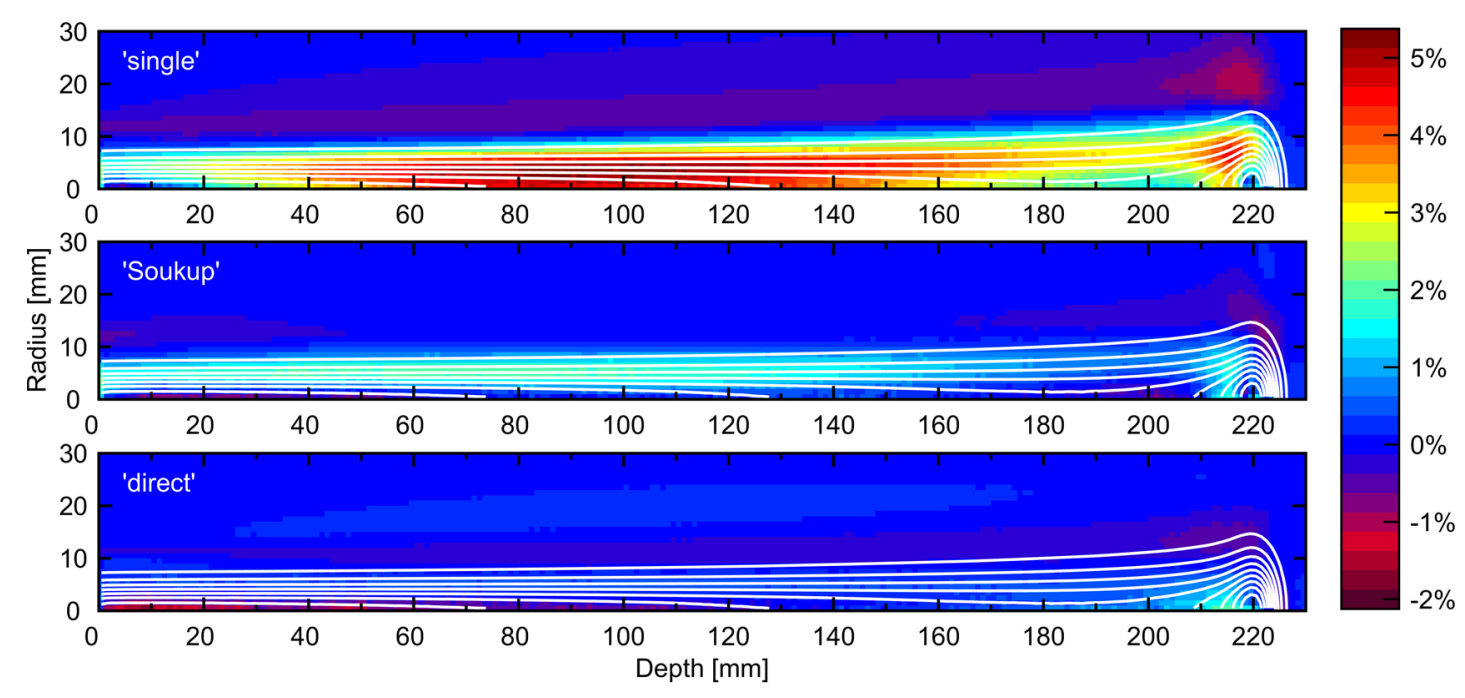

FIGURE 3 | Errors in radial distributions as a percentage of maximum dose for a PB of 220 mm BP depth when comparing the presented implementation to a MC simulation. The panels show the previously presented single-Gaussian implementation (top), the Soukup model (middle), and the direct model (bottom). The contours show the MC isodose curves, with each line corresponding to a multiple of $10 \%$ of the max dose. 

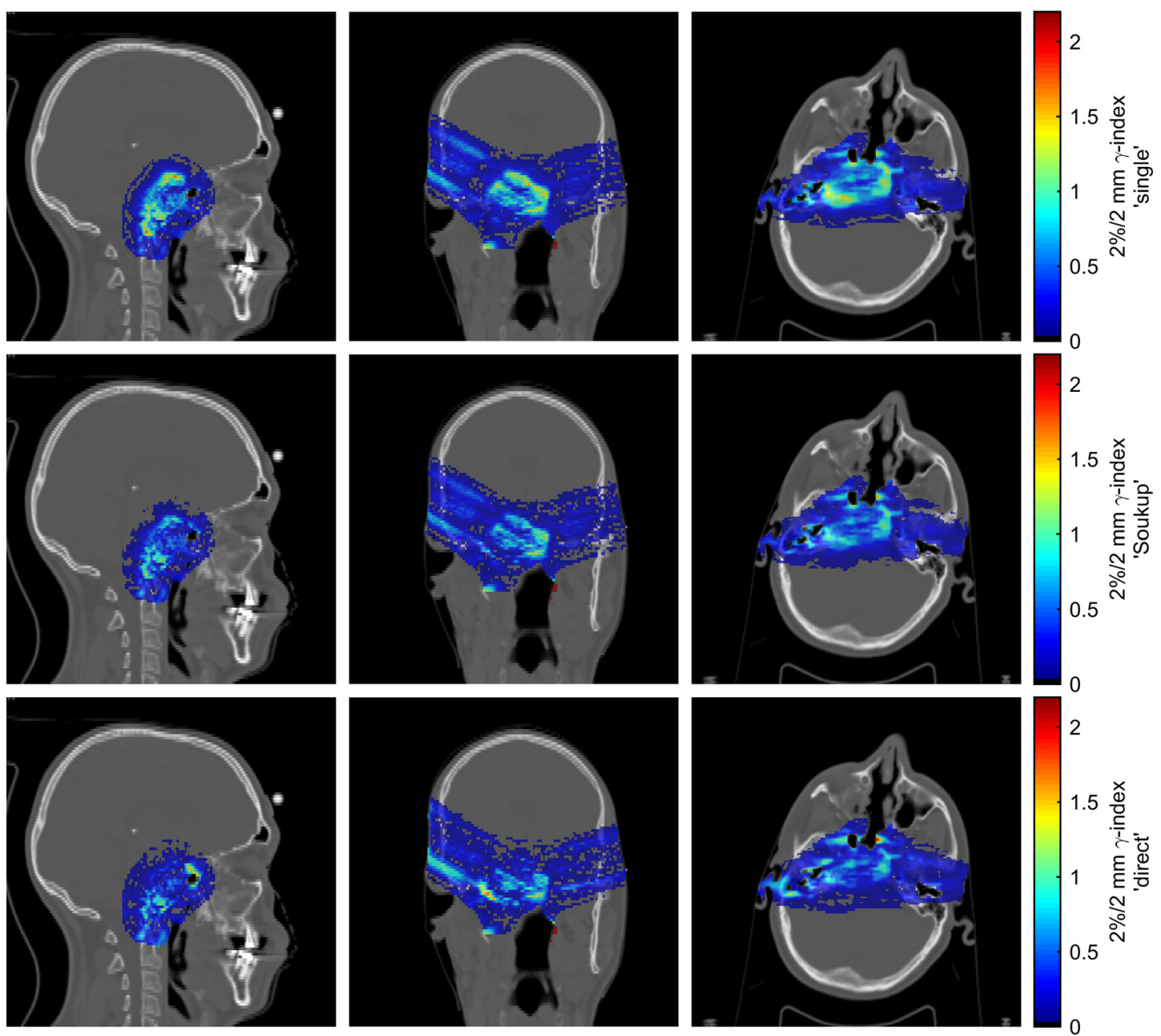

FIGURE 4 | Maps of the $\mathbf{2} \% / \mathbf{2} \mathbf{~ m m ~} \boldsymbol{\gamma}$-index for the patient case for different beam model parametrizations. The rows correspond, from top to bottom, to the single-Gaussian, Soukup, and direct models. Columns, from left to right, show sagittal, coronal, and axial slices roughly through the center of the target.

for an energy layer was 3.2 and $3.3 \mathrm{~ms}$ for the two models, or about $50 \%$ longer than for the single-Gaussian model, whereas the longest calculation time was 8.1 and $8.4 \mathrm{~ms}$, or around 25\% longer than for the single-Gaussian beam model. The overall increase in calculation time was slightly larger for the deeper test case consisting of a cubic target in water, which required $153 \mathrm{~ms}$ using the Soukup model and $157 \mathrm{~ms}$ using the direct model, which is 13 and $16 \%$ longer than the $135 \mathrm{~ms}$ required by the single-Gaussian model. The calculation times for the shallowest energy layer were 7.4 and $7.6 \mathrm{~ms}$, and for the deepest energy layer 16.5 and $16.8 \mathrm{~ms}$. Compared to the 6.0 and $13.3 \mathrm{~ms}$ required by the single-Gaussian beam model for the shallowest and deepest energy layer, this corresponds to an increase of roughly $25 \%$ in both cases.

\section{DISCUSSION}

\section{Validity of Approach}

The reason for including two parameterizations of the double-Gaussian beam model was primarily to investigate their effect on the calculation time. Consequently, the tuning of the beam models implemented was kept as simple as possible, without much of the time-consuming and detailed analysis that is associated with the commissioning of a clinical dose calculation engine. Therefore, the result obtained using the presented models may not be representative of the selected algorithm or the parameterization methods themselves, other than to serve as a lower bound for their accuracy. On the contrary, smaller errors can be expected for both models if, for example, model-specific tuning or energy-dependent tuning were used, as discussed in the following subsection. The two models are, however, expected to capture the essence of the two main types of parameterizations, namely, those aimed specifically at modeling the contributions from nuclear interactions and those based on direct fitting of lateral profiles (which thus also include other contributions to the halo). Therefore, the presented parameterizations should be indicative of how sensitive the performance of the implementation is to the model used. 
It should be pointed out that in the original implementation (12), the dose from CPBs and HPBs was calculated directly in the global dose grid. Therefore, the lack of sub-PB splitting for the halo dose served only to limit the number of HPBs but did not reduce the resolution used in the KS step. However, using a single HPB per PB already limits the effective resolution of the halo calculation. Therefore, when the KS is carried out in $\mathrm{BEV}$ coordinates as here, using the same reduced resolution also in the KS step should not affect the accuracy of the calculation, provided that the kernel varies slowly across the $\mathrm{BEV}$ voxels. Since, compared to the CPBs, the HPB kernel widths are increased by a similar amount to the voxel spacing, this should hold also for the HPBs. Thus, the effect of the lower resolution in the KS step is not expected to affect the accuracy of the calculation noticeably.

\section{Beam Model Parameterizations}

Since the Soukup model was implemented directly from the analytical expressions given in Eqs 3 and 4, little room was left for adjustments of the parameterization itself. Despite this, it resulted in a clear improvement over the single-Gaussian model both in the dose for a single PB in Figure 3 and in the $2 \% / 2 \mathrm{~mm} \gamma$-index passing rates in the patient case. The overall improvement in the $\gamma$-indices compared to the single-Gaussian beam model can also be seen in Figure 4. Still, the model assumes that there is no halo dose at the surface (c.f. Figure 2), whereas the MC results shown in Figure 1 clearly suggest the presence of such a contribution across the therapeutic range of energies for the beam line considered in this work. Therefore, using a more accurate description of the beam profile in air, such as a sum of two Gaussians, would likely further reduce the errors in the plateau region. A simple version of such an improvement would affect only how the weights are distributed between CPBs, and thus would have a negligible effect on the calculation time.

Although the direct parameterization method showed the best overall agreement for the single PB in water in Figure 3, the agreement was slightly worse than for the ideal fits of a sum of two Gaussians seen in Figure 1. The main reason for this is thought to be that, in order to more accurately model the multiple scattering in beams passing through different materials, the $\mathrm{CPB}$ widths of the primary contribution were calculated, as previously described (7), rather than obtained directly from the fit in water. Therefore, the width of the primary contribution at any given depth is constrained by the parameters $E_{S}$ and $\delta$, and, contrary to the halo contribution, cannot vary arbitrarily. More interestingly, the better average agreement did not translate to the $\gamma$-index passing rates, where the Soukup model showed better results. Looking at Figure 4, the $\gamma$-indices for these two models display similar behavior except for in limited regions where the indices for the direct model are considerably higher (c.f. the lower part of the left field in the coronal view of Figure 4). The reasons for this are not entirely clear. One explanation could be the relatively large underestimation of the $\mathrm{PB}$ central axis dose to a small number of voxels close to the surface seen in Figure 3. Another could be the larger halo fraction, which excludes more than just the nuclear interaction products from the more accurate physical modeling of the primary contribution. A third might be the rather arbitrary reduction of the width of the $\mathrm{PB}$ entrance profile that was employed to make the direct parameterization compatible with the existing beam model in air. In an improvement of the direct parameterization, constraints set on the primary contribution by $E_{\mathrm{S}}$ and $\delta$ could thus be included already in the fitting of the sum of two Gaussians. Furthermore, since the fit was seen to be relatively flexible, a preference to limit the size of the halo fraction could be included in order not to remove too much of the weight from the primary contribution. Finally, the empirical shrinking of the entrance dose applied here could be more accurately incorporated in the description of the beam profile in air.

\section{Calculation Times}

The benchmarking showed that, using one HPB per physical PB, the incorporation of a double-Gaussian beam model into the presented dose calculation engine lead to an increase in the total calculation time of no more than $16 \%$ for the two treatment plans tested. The increase in calculation time was larger for individual energy layers, ranging from about $50 \%$ for a small, shallow energy layer to around $25 \%$ for energy layers large enough to saturate the GPU. Both in the case of complete treatment plans and single energy layers, the increase in calculation time varied by only a few percentage points between the two parameterization models tested. These findings have two major consequences. The first is that employing either of the investigated parameterizations of the double-Gaussian beam model does not impact upon the suitability of the presented dose calculation engine for use in online dose calculation applications; calculation times of 16.5-16.8 ms for the deepest energy layer of the presented plans are still considerably shorter than the time between energy layers or the duration of a typical motion phase. The second is that as long as the presented implementation of the double-Gaussian beam model is used, the calculation time is unlikely to change significantly for other, beam line-specific or more sophisticated, parameterizations of the halo dose. Together, these indicate that, using a single GPU, it is possible to achieve fast enough calculation times for online dose calculation while maintaining the same accuracy as a widely adopted clinical algorithm, independent of the specific beam line.

The larger increase in calculation time for single energy layers than for complete treatment plans can be explained by the varying fractions of the total calculation time spent on different steps of the calculation. The calculation of complete treatment plans was dominated by the KS step, which, using the single-Gaussian beam model, was responsible for $76 \%$ of the calculation time for the patient plan and $88 \%$ of the calculation time for the cubic test case (excluding the time spent on memory transfers in both cases). Using the double-Gaussian parameterizations, the increase in calculation time for the KS step was 3-5\% for the patient case and $14-18 \%$ for the cubic test case, which therefore resulted in increases of a similar order in the total calculation time for entire plans. For the individual energy layers, on the other hand, where the KS step is carried out only once, the calculation times of the steps that are carried out once per beam direction become comparable to the KS time. The calculation times for these steps generally increased more than that for the KS step when going from the single-Gaussian to the double-Gaussian beam model. In particular, the time required to set up the calculation and allocate 
memory for BEV intermediates and the time to copy the dose distribution to texture memory both increased by between 20 and $40 \%$. Furthermore, due to the larger number of voxels reached by the halo, the time required to transform the dose from the BEV coordinate system to the global dose grid roughly doubled. In the light of this, the overall increases in calculation time of $50 \%$ for a small energy layer or $25 \%$ for large energy layers are not surprising.

\section{CONCLUSION}

We have described how a double-Gaussian beam model was incorporated into an existing implementation of the PB algorithm running on a GPU while avoiding the prohibitive increase in calculation time expected from the large halo width. The increase in calculation time was not larger than $16 \%$ for entire treatment plans, and about $25 \%$ for large energy layers, which are the most time-consuming to calculate. Therefore, the addition of a double-Gaussian beam model does not alter the suitability of the presented implementation for use in online dose calculation. The calculation time was further shown to be relatively unaffected by the specific parameterization used to describe the halo dose contribution. Despite the calculation of the halo contribution being simplified compared to that of the primary, it was based on the same algorithm as a widely used commercial TPS. Therefore, it is expected that with adequate tuning, it will be able to reproduce with sufficient accuracy the halo dose of a general beam line. Based on these observations, we conclude that, using

\section{REFERENCES}

1. JiaX,Ziegenhein P, Jiang SB. GPU-based high-performance computing for radiation therapy. Phys Med Biol (2014) 59:R151-82. doi:10.1088/0031-9155/59/4/ R151

2. Yepes PP, Mirkovic D, Taddei PJ. A GPU implementation of a track-repeating algorithm for proton radiotherapy dose calculations. Phys Med Biol (2010) 55:7107-20. doi:10.1088/0031-9155/55/23/S11

3. Kohno R, Hotta K, Nishioka S, Matsubara K, Tansho R, Suzuki T. Clinical implementation of a GPU-based simplified Monte Carlo method for a treatment planning system of proton beam therapy. Phys Med Biol (2011) 56:287-94. doi:10.1088/0031-9155/56/22/N03

4. Jia X, Schümann J, Paganetti H, Jiang SB. GPU-based fast Monte Carlo dose calculation for proton therapy. Phys Med Biol (2012) 57:7783-97. doi:10.1088/0031-9155/57/23/7783

5. Giantsoudi D, Schuemann J, Jia X, Dowdell S, Jiang S, Paganetti H. Validation of a GPU-based Monte Carlo code (gPMC) for proton radiation therapy: clinical cases study. Phys Med Biol (2015) 60:2257. doi:10.1088/0031-9155/60/6/2257

6. Tseung HWC, Ma J, Beltran C. A fast GPU-based Monte Carlo simulation of proton transport with detailed modeling of nonelastic interactions. Med Phys (2015) 42:2967-78. doi:10.1118/1.4921046

7. Da Silva J, Ansorge R, Jena R. Sub-second pencil beam dose calculation on GPU for adaptive proton therapy. Phys Med Biol (2015) 60:4777-95. doi:10.1088/0031-9155/60/12/4777

8. Da Silva J, Ansorge R, Jena R. Efficient scatter-based kernel superposition on GPU. J Parallel Distrib Comput (2015) 84:15-23. doi:10.1016/j. jpdc.2015.07.003

9. Lax I, Brahme A. Electron beam dose planning using Gaussian beams energy and spatial scaling with inhomogeneities. Acta Oncol (1985) 24:75-85. doi:10.3109/02841868509134369

10. Russell KR, Isacsson U, Saxner M, Ahnesjö A, Montelius A, Grusell E, et al. Implementation of pencil kernel and depth penetration algorithms a single GPU, dose distributions from individual energy layers can be calculated with comparable accuracy to a modern clinical TPS well within the time of a typical motion phase or change of beam energy.

\section{AUTHOR CONTRIBUTIONS}

JS, RA, and RJ contributed to the conception and design of the study, and JS was responsible for the acquisition and analysis of the data. JS drafted the work that was critically revised for important intellectual content by RA and RJ. All authors approve of the submitted version and agree to be accountable for all aspects of the work.

\section{ACKNOWLEDGMENTS}

We would like to thank Mario Ciocca, Giuseppe Magro, Andrea Mairani, and Silvia Molinelli at CNAO (Pavia, Italy) for sharing data and models of the CNAO beam line, and for providing the patient case and corresponding dose distribution calculated with Fluka. We would further like to thank Till Böhlen at MedAustron (Wiener Neustadt, Austria) for help with MC simulations of individual PBs. This research was funded by the European Commission Seventh Framework People Programme through the ENTERVISION project, grant agreement 264552. RJ is funded in part by Cancer Research UK, grant number 13716. The Tesla K40 GPU used for benchmarking was donated by the Nvidia Corporation through their Hardware Grant Program.

for treatment planning of proton beams. Phys Med Biol (2000) 45:9-27. doi:10.1088/0031-9155/45/1/302

11. Pedroni E, Scheib S, Böhringer T, Coray A, Grossmann M, Lin S, et al. Experimental characterization and physical modelling of the dose distribution of scanned proton pencil beams. Phys Med Biol (2005) 50:541-61. doi:10.1088/0031-9155/50/3/011

12. Soukup M, Fippel M, Alber M. A pencil beam algorithm for intensity modulated proton therapy derived from Monte Carlo simulations. Phys Med Biol (2005) 50:5089-104. doi:10.1088/0031-9155/50/21/010

13. Sawakuchi GO, Titt U, Mirkovic D, Ciangaru G, Zhu XR, Sahoo N, et al. Monte Carlo investigation of the low-dose envelope from scanned proton pencil beams. Phys Med Biol (2010) 55:711-21. doi:10.1088/0031-9155/55/3/011

14. Schwaab J, Brons S, Fieres J, Parodi K. Experimental characterization of lateral profiles of scanned proton and carbon ion pencil beams for improved beam models in ion therapy treatment planning. Phys Med Biol (2011) 56:7813-27. doi:10.1088/0031-9155/56/24/009

15. Ulmer W, Schaffner B. Foundation of an analytical proton beamlet model for inclusion in a general proton dose calculation system. Radiat Phys Chem (2011) 80:378-89. doi:10.1016/j.radphyschem.2010.10.006

16. Clasie B, Depauw N, Fransen M, Gomà C, Panahandeh HR, Seco J, et al. Golden beam data for proton pencil-beam scanning. Phys Med Biol (2012) 57:1147-58. doi:10.1088/0031-9155/57/5/1147

17. Parodi K, Mairani A, Sommerer F. Monte Carlo-based parametrization of the lateral dose spread for clinical treatment planning of scanned proton and carbon ion beams. J Radiat Res (2013) 54:i91-6. doi:10.1093/jrr/rrt051

18. Ciangaru G, Sahoo N, Zhu XR, Sawakuchi GO, Gillin MT. Computation of doses for large-angle Coulomb scattering of proton pencil beams. Phys Med Biol (2009) 54:7285-300. doi:10.1088/0031-9155/54/24/003

19. Sawakuchi GO, Zhu XR, Poenisch F, Suzuki K, Ciangaru G, Titt $\mathrm{U}$, et al. Experimental characterization of the low-dose envelope of spot scanning proton beams. Phys Med Biol (2010) 55:3467-78. doi:10.1088/0031-9155/55/12/013 
20. Fuchs H, Ströbele J, Schreiner T, Hirtl A, Georg D. A pencil beam algorithm for helium ion beam therapy. Med Phys (2012) 39:6726-37. doi:10.1118/1.4757578

21. Li Y, Zhu RX, Sahoo N, Anand A, Zhang X. Beyond Gaussians: a study of single-spot modeling for scanning proton dose calculation. Phys Med Biol (2012) 57:983-97. doi:10.1088/0031-9155/57/4/983

22. Zhu X, Poenisch F, Lii M, Sawakuchi G, Titt U, Bues M, et al. Commissioning dose computation models for spot scanning proton beams in water for a commercially available treatment planning system. Med Phys (2013) 40:041723. doi:10.1118/1.4798229

23. Bellinzona V, Ciocca M, Embriaco A, Fontana A, Mairani A, Mori M, et al. On the parametrization of lateral dose profiles in proton radiation therapy. Phys Med (2015) 31:484-92. doi:10.1016/j.ejmp.2015.05.004

24. Gottschalk B, Cascio EW, Daartz J, Wagner MS. On the nuclear halo of a proton pencil beam stopping in water. Phys Med Biol (2015) 60:5627-54. doi:10.1088/0031-9155/60/14/5627
25. Ferrari A, Sala PR, Fasso A, Ranft J. FLUKA: A Multi-Particle Transport Code. Menlo Park, CA: Stanford Linear Accelerator Center (SLAC) (2005).

26. Böhlen TT, Cerutti F, Chin MPW, Fassò A, Ferrari A, Ortega PG, et al. The FLUKA code: developments and challenges for high energy and medical applications. Nucl Data Sheets (2014) 120:211-4. doi:10.1016/j.nds.2014.07.049

Conflict of Interest Statement: The authors declare that the research was conducted in the absence of any commercial or financial relationships that could be construed as a potential conflict of interest.

Copyright $\odot 2015$ da Silva, Ansorge and Jena. This is an open-access article distributed under the terms of the Creative Commons Attribution License (CC BY). The use, distribution or reproduction in other forums is permitted, provided the original author(s) or licensor are credited and that the original publication in this journal is cited, in accordance with accepted academic practice. No use, distribution or reproduction is permitted which does not comply with these terms. 